\title{
ON THE RELATIVISTIC GRAVITATIONAL COLLAPSE ET CETERA
}

\author{
Nota del m.e. ANGELO LOINGER (*) e di TIZIANA MARSICO (**)
}

(Adunanza del 23 ottobre 2014)

SuNTO. - Sfere di "polvere" di grande massa collassano gravitazionalmente in "sfere piene" compatte di volumi finiti, le cui superfici hanno le medesime proprietà dell'orizzonte degli eventi associato alla gravitazione di un punto materiale. Sia l'orizzonte degli eventi di una "sfera piena" sia quello associato ad un punto materiale, in virtù della repulsione gravitazionale hilbertiana non possono "inghiottire" alcunché, contrariamente a quanto afferma un locus communis. I dati osservazionali convalidano i nostri risultati.

$* * *$

ABSTRACT. - Massive and supermassive "dust" spheres (with a zero internal pressure) collapse to compact "full spheres" of finite volumes, whose surfaces have the properties of the event horizon of a gravitating mass-point. By virtue of Hilbert's repulsive effect, both the event horizon of a mass-point and the event horizon of a "full sphere" cannot "swallow" anything, in contradiction with the assertion of a locus communis. The observational data corroborate our results.

1. - The main theme of this paper is treated in sect. 5. In sect. 2 we recall a general expression (de Sitter, Eddington, Levi-Civita) of the

(*) Dipartimento di Fisica, Università degli Studi di Milano, Italy.

E-mail: angelo.loinger@mi.infn.it

(*) Liceo Classico “G. Berchet”, Via della Commenda 26, 20122 Milano, Italy.

E-mail: martiz64@libero.it 
metric of a Schwarzschild spacetime created by a gravitating masspoint. Sects. 3, 3bis, 4 give a concise formulation of the Hilbertian gravitational repulsion, which is the essential instrument for the developments of sect. 5. In Appendix some remarks on past and present things.

2. - In 1926 Levi-Civita [1] gave a geometrically explicit explanation of the general form of solution (de Sitter, Eddington) to the Schwarzschild problem to find the Einsteinian field created by a gravitating point-mass $M$ at rest. He adopted a Palatini's method [2], which yields the appropriate geometrical definition of spherical symmetry in a curved spatial manifold, and the justification of the employment in it of the polar coordinates $r(\geq 0), \vartheta(0 \leq \vartheta \leq \pi), \varphi(0 \leq \varphi<2 \pi)$. He found, with de Sitter and Eddington:

$\mathrm{d} s^{2}=\left[1-\frac{2 m}{\mathcal{R}(r)}\right] c^{2} \mathrm{~d} t^{2}-\left[1-\frac{2 m}{\mathcal{R}(r)}\right]^{-1}[\mathrm{~d} \mathcal{R}(r)]^{2}-[\mathcal{R}(r)]^{2}\left(\mathrm{~d} \vartheta^{2}+\sin ^{2} \vartheta \mathrm{d} \varphi^{2}\right)$,

where: $m \equiv G M / c^{2}$, and $\mathcal{R}(r)$ is any regular function of $r$, which gives a Minkowskian $\mathrm{d} s^{2}$ at $r=\infty$. For $\mathcal{R}(r)=r$ we have the standard (Hilbert, Droste, Weyl) form of solution; for $\mathcal{R}(r)=\left[r^{3}+(2 m)^{3}\right]^{1 / 3}$ and $\mathcal{R}(r)=r+$ $2 m$ the original Schwarzschild's [3] and Brillouin's [4] forms of solution, respectively. (Remark that the forms [3] and [4] are maximally extended - and thus the baroque known form of solution by Kruskal and Szekeres is quite superfluous).

It is evident from Levi-Civita's treatment that eq. (1) has a mathematical and physical meaning only for $\mathcal{R}(r)>2 m$, and that no role inversion between $\mathcal{R}(r)$ and $t$ for $\mathcal{R}(r) \leq 2 m$ is allowed.

Temporarily forgetting that when $\mathcal{R}(r) \leq 2 m$, metric (1) loses any meaning, we could claim that the surface area $A=4 \pi(2 m)^{2}$ represents an invariant and significant notion - and the so-called "Schwarzschild radius" $2 m$ is physically meaningful. But this forgetting is not permitted, and we understand why the Founding Fathers of general relativity (GR) rejected the idea to give a physical meaning to the "globe" $\mathcal{R}(r) \leq 2 \mathrm{~m}$. As a matter of fact, the astrophysical phenomena that have been interpreted as originated by a "globe" of this kind can be plainly interpreted as due to a great, or enormous, mass concentrated in a relatively small space region. In particular, no "swallowing" property of the event borizon $\mathcal{R}(r)=2 m$ has ever been observed. (Remark that the radially moving test-particles and light-rays arrive at $\mathcal{R}(r)=2 m$ with zero velocity and zero acceleration). Kundt [5] thinks that the stellar-mass "globe" - candidates are in 
reality neutron stars inside massive accretion disks, and that the central engine of an AGN (active galactic nucleus) is a nuclear-burning disk.

3. - We have treated in several papers [6] various consequences and applications of the Hilbertian gravitational repulsion [7]. Here, we limit ourselves to expose the essential of this concept.

First of all, we recall the fundamental equations of the radial geodesics of Schwarzschild manifold created by a mass-point. Putting with Hilbert $2 m \equiv \alpha$, we obtain from eq. (1) - see Hilbert [7] (with $r$ in lieu of $\mathcal{R}(r):=\mathcal{R})$ :

$$
\begin{aligned}
& \frac{\mathrm{d}^{2} \mathcal{R}}{c^{2} \mathrm{~d} t^{2}}-\frac{3 \alpha}{2 \mathcal{R}(\mathcal{R}-\alpha)}\left(\frac{\mathrm{d} \mathcal{R}}{c \mathrm{~d} t}\right)^{2}+\frac{\alpha(\mathcal{R}-\alpha)}{2 \mathcal{R}^{3}}=0 ; \\
& \left(\frac{\mathrm{d} \mathcal{R}}{c \mathrm{~d} t}\right)^{2}=\left(1-\frac{\alpha}{\mathcal{R}}\right)^{2}+A\left(1-\frac{\alpha}{\mathcal{R}}\right)^{3},
\end{aligned}
$$

where the constant $A$ of first integral (3) is negative for the test-particles and zero for the light-rays. We put $A=-|A|$.

Eq. (2) tells us that the acceleration is negative or positive - i.e., that the gravitation acts in an attractive or in a repulsive way - according to the absolute value of the velocity: when

$$
\left|\frac{\mathrm{d} \mathcal{R}}{c \mathrm{~d} t}\right|<\frac{1}{\sqrt{3}}\left(1-\frac{\alpha}{\mathcal{R}}\right)
$$

we have attraction; but when

$$
\left|\frac{\mathrm{d} \mathcal{R}}{c \mathrm{~d} t}\right|>\frac{1}{\sqrt{3}}\left(1-\frac{\alpha}{\mathcal{R}}\right)
$$

we have repulsion.

Let us call $\mathcal{R}_{*}$ the value of $\mathcal{R}$ such that $\mathrm{d}^{2} \mathcal{R} / c^{2} \mathrm{~d} t^{2}=0$ : attraction and repulsion counterbalance each other. At $\mathcal{R}=\mathcal{R} *$ the absolute value of the velocity $|\mathrm{d} R / c \mathrm{~d} t|$ is maximal:

$$
\left|\frac{\mathrm{d} \mathcal{R}}{c \mathrm{~d} t}\right|_{\max }=\frac{1}{\sqrt{3}}\left(1-\frac{\alpha}{\mathcal{R}_{*}}\right) \quad ; \quad \mathcal{R}_{*}=\frac{3|A| \alpha}{3|A|-2} .
$$

For the light-rays $A=0$; eq. (3) gives

$$
\left|\frac{\mathrm{d} \mathcal{R}}{c \mathrm{~d} t}\right|=1-\frac{\alpha}{\mathcal{R}}:
$$


the light is repulsed everywhere; $|\mathrm{d} \mathcal{R} / \mathrm{d} t|$ increases from zero at $\mathcal{R}=\alpha$ to $c$ at $\mathcal{R}=\infty$.

It follows from the above equations that the test-particles and the light- rays arrive at $\mathcal{R}=\alpha$ with zero velocity and zero acceleration - as we have anticipated in sect. 2 . The spatial surface $\mathcal{R}=\alpha$ represents for them an insuperable barrier: a fact of paramount importance from the astrophysical standpoint. In other terms, we can affirm that the event borizon $\mathcal{R}=\alpha$ is "inappetent".

We see that attraction and repulsion are linked in a physical way to the three -acceleration and the three-velocity. This means that the physical evolution-parameter is the "Systemzeit" $t$ (von Laue) of Schwarzschild spacetime, not the proper time of the particles or the affine parameter of the light-rays, as we shall prove in the following sect. 3 bis.

3bis. - There exists a tentative to give a meaning to space region $\mathcal{R} \leq \alpha$ : with the substitutions $\mathcal{R} \rightarrow \alpha-c t$ and $c t \rightarrow \alpha-\mathcal{R}$, eq. (1) becomes:

$$
\left(\mathrm{d} s^{2}\right)_{\mathcal{R} \leq \alpha}=\left(\frac{\alpha-c t}{c t}\right) c^{2} \mathrm{~d} t^{2}-\left(\frac{c t}{\alpha-c t}\right) \mathrm{d} \mathcal{R}^{2}-(\alpha-c t)^{2}\left(\mathrm{~d} \vartheta^{2}+\sin ^{2} \vartheta \mathrm{d} \varphi^{2}\right)
$$

thus, a static problem has become non-static in a limited spatial region. In particular: at $\mathcal{R}=\alpha$ we have $t=0$, at $\mathcal{R}=0$ we have $t=\alpha / c$. We see that eq. (1') describes an absurd physical situation. Further, the metrics (1) and (1') are not continuously connected. We can also remark that the transition to a time-dependent problem implies the introduction of inertial forces, that are completely extraneous to the real problem.

We emphasize finally that also the famous metric of Kruskal and Szekeres is non-static, and therefore introduces inertial forces in a static situation.

It follows immediately from the Schwarzschildian equations of the radial geodesics that, both for particles and light-rays, $(\mathrm{d} \mathcal{R} / \mathrm{d} p)^{2}$ is different from zero ( $p$ is an affine evolution-parameter) at $\mathcal{R}=\alpha$.

For the particles also $(\mathrm{d} \mathcal{R} / \mathrm{d} s)^{2}$ is different from zero at $\mathcal{R}=\alpha$. it seems that the event horizon $\mathcal{R}=\alpha$ has become "appetent". But this conclusion is misleading, because - as we have seen - no reasonable metric exists in $\mathcal{R} \leq \alpha$.

A last remark. It was observed by von Laue [8] that the coordinate-time $t$ of Schwarzschild manifold of a gravitating mass-point has 
the character of a physical "Systemzeit", as it is proved by its role in the explanations of the red-shifts of the spectral lines.

4. - For the Schwarzschildian circular geodesics Hilbert's gravitational repulsion acts in a simple way. For the particles we have the following inequalities:

$$
\begin{aligned}
& \mathcal{R}>\frac{3}{2} \alpha ; \\
& v \equiv \mathcal{R} \frac{\mathrm{d} \varphi}{\mathrm{d} t}<\frac{c}{\sqrt{3}} .
\end{aligned}
$$

Remark that $v^{2}=\alpha c^{2} / 2 \mathcal{R}$, as in Newton theory.

For the light-rays $(A=0)$ we have two equalities:

$$
\begin{aligned}
& \mathcal{R}=\frac{3}{2} \alpha ; \\
& v=\frac{c}{\sqrt{3}} .
\end{aligned}
$$

The restrictions (8), (9), (10), (11) are not deducible from the geometric differential equation that connects directly $\mathcal{R}$ and $\varphi$. (See Appendix A of paper [9].)

5. - According to well-known - but not irresistible arguments - a cold, degenerate star, whose mass is equal to $\approx(3 \pm 1)$ solar masses, and beyond, collapses inevitably and forms a "globe" $\mathcal{R}(r)=r=2 m$. And since in GR a fluidodynamical pressure is not weightless, its presence would accelerate the gravitational collapse.

However, McVittie [10] and the present authors [11] have proved with exact computations that a spherically-symmetric gravitational collapse, under the action of a time-dependent pressure $p(t)$, ends up in a body with a finite extent.

We shall now demonstrate a very general result: even with a zero pressure, by virtue of the decisive action of the Hilbertian repulsive effect, a spherically-symmetric collapse of a body composed of "dust" particles ends up in a final stage with a comparatively small, finite, volume. 
Of course, the particles will describe geodesic lines. The interval $\mathrm{d} s_{\text {ext }}$ outside the sphere is given by eq. (1).

In Newton theory the gravitational collapse of a "dust' model, whose particles are distributed with spherical symmetry about a centre, ends in a material point with an infinite mass density in a finite time.

But in GR things go otherwise. As in Newton theory, the surface shell of the "dust" sphere contracts itself as if the total mass $M=m c^{2} / G$ were concentrated in the centre. The other concentric spherical shells contract themselves as if the mass in the centre were correspondently reduced. By virtue of the Hilbertian repulsion, the particles of the superficial shell arrive on the surface whose area is equal to $4 \pi(2 m)^{2}$ with a zero velocity $\mathrm{d} \mathcal{R} / \mathrm{d} t$ and a zero acceleration $\mathrm{d}^{2} \mathcal{R} / \mathrm{d} t^{2}$. The particles of a generic shell arrive with zero velocity and zero acceleration on the spherical surface whose area is equal to $4 \pi[2 \cdot \text { (the pertinent partial mass) }]^{2}$.

Evidently, the collapse ends when the total mass $M$ of the star has filled up the sphere whose superficial area is $4 \pi(2 m)^{2}$.

We see that the famous arguments, that we have above mentioned, according to which the collapse of the "dust" sphere ends with the formation of the "globe" $r=2 m$, with its "hard" singularity at $r=0$ and its "soft" singularity at $r=2 m$, do not describe the real physical situation.

Two final remarks. $i$ ) It is clear that the event horizon $\mathcal{R}=2 m$ of the above "full sphere" - like the event horizon of a "globe" - does not "swallow" anything. ii ) As it was proved by Schwarzschild, there is a minimal radius, equal to $(9 / 8) \cdot(2 m)$, for an incompressible fluid sphere with a given mass [12].

\section{APPENDIX - REMARKS ON PAST AND PRESENT THINGS}

In the previous treatment we have designed (with M. Brillouin) the spatial region $\mathcal{R} \leq 2 m$ as a "globe". Its current name is "black hole" $(\mathrm{BH})$, because with a widespread, but erroneous, interpretation one assigns to it the following characteristic properties - expressed with the radial coordinate $\mathcal{R}(r)=r$ : the space $r<2 m$ cannot communicate with the space in which $r>2 m$; any signal, even a light signal, would take an infinite time to cross the boundary $r=2 \mathrm{~m}$. Thus one cannot have a direct observational knowledge of the region $r<2 m$; such a region is called a "black hole", because things may fall into it, taking an infinite time, but nothing can come out. 
Now, the "proof" of these miraculous properties rests on a paralogism: indeed, if one bides (as many authors do, in particular Lémaittre, Kruskal and Szekeres) the "soft" singularity $r=2 m$ in the connection between the coordinates $(r, t)$ and suitable new coordinates $(\varrho, \tau)$, it is possible to extend the transformed solution (which is non-static!) to the region $r<2 m$, thus "justifying" in particular the interchange in it of the roles of $r$ and $t$. (In $r=0$ we have a "hard" singularity).

Einstein, Levi-Civita, Schwarzschild, Hilbert, Weyl, Eddington, Pauli, von Laue, Fock, ..., who created and developed the relativity theory always rejected the very notion of BH. In 1939 Einstein [13] investigated a dynamical model composed of several particles, and demonstrated that its behaviour is such that no $\mathrm{BH}$ of the ensemble can appear. However, since the Sixties of the past century lots of papers have been published with the aim of exhibiting the wonderful properties of the BHs. And the astrophysical community made a bistorical falsification by claiming that the mathematical structure of GR had been deeply understood only in the mentioned Sixties. On the contrary, the concepts of the modern differential geometry have been created in the Thirties; better, in the Twenties, if we take into account some papers by Weyl. Furthermore, with another historical falsification it was claimed that Schwarzschild and Einstein foresaw the existence of BHs.

A widespread "Vulgate" of GR claims that if the problem of the Einsteinian field created by a gravitating point-mass is solved using coordinate-free methods (as orthonormal bases, etc.), the result is necessarily the standard form of solution, for which $R=r$. But the procedure of the "Vulgate" is impaired by a vulgar fallacy: in it the angular part is written $r^{2}\left(\mathrm{~d} \vartheta^{2}+\sin ^{2} \vartheta\right)$, and with this postulate the standard form of solution is already chosen.

It is useful to recall that for $r \geq 0$ the forms of solution by Schwarzschild $\left(\mathcal{R}(r)=\left[r^{3}+(2 m)^{3}\right]^{1 / 3}\right)$ and by Brilluoin $(\mathcal{R}(r)=r+2 m)$ are diffeomerphic to the standard form (Hilbert, Droste, Weyl) for $r \geq 2 m$.

Physics is essentially an experimental and observational science. It seems to us that the astrophysical community has neglected this fact with the introduction of fictive notions as the black holes, the gravitational waves, and several other things. A New Scholasticism has been created, which ostracizes the scientists who abhor its bad metaphysics.

(An amazing instance of bad metaphysics is the purpose to investigate the interaction of two BHs. Now, there exists no solution of Einstein field equations, which describes two BHs. In numerical rela- 
tivity, the authors work out some expressions that they think apt to describe the approximate behaviour of a pair of interacting BHs.)

In a paper of 2008 [14] entitled "On the radial geodesics of Kerr's manifold" we have demonstrated the following result: the event horizons of the famous Kerr's manifold are incapable of "swallowing" anything, because the "stationary-limit" surface, which is external to the event horizons, exerts a gravitational repulsion $\left(\mathrm{d} r / \mathrm{d} t=0 ; \mathrm{d}^{2} r / \mathrm{d} t^{2} \geq 0\right)$ on the arriving particles and light-rays, that is quite similar to the Schwarzschildian repulsive action.

In a paper entitled "The event horizon of Sagittarius $A^{*}$ " [15], the authors affirm that recent millimetric and infrared observations of the supermassive centre $\operatorname{Sgr} A^{*}$ of the Milky Way require the existence of a central BH. Now, we have demonstrated (see sect. 5) that the final stage of the collapse of a massive or supermassive "dust" sphere is a compact "full sphere" of a relatively small, but finite, volume. In the paper quoted in [16] we have shown that the observational data of [15] are perfectly explained by the existence of a central "full sphere" of the above kind.

A unpleasant consequence of the erroneous notion of $\mathrm{BH}$ is the belief in the so-called "Information Paradox", from which it would follow that GR is in contradiction with the time-reversibility. Indeed, it has been affirmed (as we have recalled in the first paragraph of this Appendix) that in the instance of Schwarzschild's manifold created by a gravitating point-mass the test particles and the light-rays go beyond the space surface $\mathcal{R}(r)=2 m$, and disappear from the "external" world for ever, with an irreversible process.

We have shown (see [17]) that if one takes into account all the assumptions which characterize the deduction of the geometric differential equation of the Schwarzschildian geodesic trajectories $[\mathcal{R}(r)=\mathrm{a}$ function of $\varphi ;(0 \leq \varphi<2 \pi)]$, one obtains a confirmation of the dynamical results (see sect. 3): the geodesics that arrive on the surface $\mathcal{R}(r)=$ $2 m$ find here their end: the "Information Paradox" does not exist. - An interesting by-product of [17] is as follows: the mentioned geometric equation does not yield the conditions that characterize the circular orbits. A fact which has been ignored in the previous literature.

We conclude that we could affirm a priori - i.e., without the detailed examination of the above geodesics - that the "Information Paradox" cannot have existence in a theory as the GR, which has been devised in a manner quite independent of the directions of the spacetime coordinates, in particular of the direction of the temporal coordi- 
nate - a property that was emphasized by Levi-Civita. The contrary opinion must be ascribed to a wrong interpretation of a given aspect of the formalism.

In the last decades, beyond the bad metaphysics of the BHs the Scholasticism has developed the worst metaphysics of the BHs considered from the point of view of the so-called "quantum gravity". A short digression about this locution is now useful. We give here a résumé of paper [18].

The spacetime of quantum theories are the following (as it is well known): $i$ ) the Euclidean-Newtonian substrate of the Galilean group of transformations; ii ) the Minkowskian substrate of the Lorentzian group of transformations; iii ) any given, "rigid", pseudo-Riemannian manifold. We have correspondingly: $i$ ) the nonrelativistic quantum mechanics of the dynamical systems with a finite number of degrees of freedom; $i i$ ) the Lorentzian quantum field theories - and the quantitized linearized approximation of GR (Pauli, Rosenfeld); iii ) Dirac equation for a particle in a fixed pseudo- Riemannian manifold. - The known quantum formalisms have a definite physical meaning only under the condition that the mentioned spacetimes are described by the usual non -operator quantities. Consequently, any rational quantization program of GR is doomed to a failure, because it would imply necessarily some operator characterizations of the spacetimes. But the coefficients $g_{\mu v},(\mu, v=0,1,2,3)$, of any Einsteinian metric do not represent a classical field in the conventional meaning: they "are" the spacetime. - "Quantum gravity": a patchwork of various particular concepts and results of quantum field theories (e.g., unitary evolution, CPT-theorem, etc.) acritically applied to the Einsteinian field: an enormous paralogism, which gives contradictory assertions.

Back to the "quantized BHs". For a list of many papers on this theme, see the references in [19]. In this paper we find a fictive lucubration, which contradicts all the previous fancies on the BHs. The author "proves" that the notion of an event horizon, from which nothing can escape, is incompatible with quantum theory; however, the gravitational collapse would create apparent horizons, which persist for a period of time. Thus, by virtue of a pseudo-argumentation, the author comes near to the classic, right conclusion that the $\mathrm{BHs}$ are non -existing objects.

"Die Wahrheit bildet ein System". 


\section{REFERENCES}

[1] T. Levi-Civita, The Absolute Differential Calculus (Calculus of Tensors) - (Dover Publications, Inc., Mineola, N.Y.) 2005, Chapt. XII, sects. 11, 12, 13. (First publi- shed by Blackie and Son Lim. - London and Glasgow, 1926).

[2] A. Palatini, Nuovo Cimento, 14 (1917) 12.

[3] K. Schwarzschild, Berl. Ber., (1916) 189. An English translation in arXiv:physics/9905030,(May 12 $2^{\text {th }}, 1999$.

[4] M. Brillouin, Journ. Phys. Rad., 23 (1923) 43. An English translation in arXiv:physics/0002009, February ${ }^{\text {rd }}, 2000$.

[5] W. Kundt, Astrophysics (Springer-Verlag, Berlin, etc.) 2007, p.p.190 and 187; and passim.

[6] A. Loinger and T. Marsico, arXiv:0706.3891 [physics.gen-ph] 16 Jul 2007; ibid: arXiv:0710.3927 [id.] 21 Oct 2007; ibid.: arXiv:0711.4997 [id.] 22 Dec 2007; ibid.: arXiv:0803.0050 [id.] 1 Mar 2008; ibid.: arXiv:0809.1221 [id.] 7 Sept 2008; ibid.: arXiv:0904.1578 [id.] 9 Apr 2009; ibid.: arXiv:0907.2895 [id.] 16 Jul 2009; ibid.: arXiv:0912.1329 [id.] 7 Dec 2009; ibid.: arXiv:1011.2600 [id.] 11 Nov 2010; ibid.: arXiv:1304.4190 [id.] 15 Apr 2013. See also A. Loinger and T. Marsico, "Dark Energy explained by a Schwarzschildian Cosmology - et cetera", Academia.edu and Research-gate, Mar 2014. And our preceeding Note in "Istituto Lombardo".

[7] D. Hilbert, Math. Annalen, 92 (1924) 1; also in Gesammelte Abhandlungen, Dritter Band (Springer-Verlag, Berlin) 1935, p. 258.

[8] M. v. Laue, Die allgemeiune Relativitätstheorie, 4. neubearbeitete Auflage (Friedr. Vieweg und Sohn, Braunschweig) 1956, p. 112.

[9] A. Loinger and T. Marsico, arXiv:1304.4190 [physics.gen-ph] 15 Apr 2013.

[10] G.C. Mc Vittie, Ap. J., 140 (1964) 401.

[11] A. Loinger and T. Marsico, arXiv:physics/0612160 (December 16 ${ }^{\text {th }}$, 2006).

[12] K. Schwarzschild, Berl. Ber., (1916) 424. An English translation in arXiv:physics/9912033, December $16^{\text {th }}, 1999$.

[13] A. Einstein, Ann. Math., 40 (1939) 922.

[14] A. Loinger and T. Marsico, arXiv:0809.1221 [physics.gen-ph] 7 Sep 2008. See further A. Loinger and T. Marsico, arXiv:0903.1267 [physics.gen-ph] 6 Mar 2009.

[15] A.E. Broderick et al., arXiv:0903.1105 [astro-ph, HE] 5 Mar 2009.

[16] A. Loinger and T. Marsico, arXiv:1011.2600 [physics.gen-ph] 11 Nov 2010.

[17] A. Loinger and T. Marsico, arXiv:1304.4190 [physics.gen-ph] 15 Apr 2013.

[18] A. Loinger, arXiv:physics/0308042, August 12 2003.

[19] S.W. Hawking et al., arXiv:1401.5761 [hep-th] 22 Jan 2014. 